\title{
Broadband nuclear emission in two radio-loud broad absorption line quasars ${ }^{\star}$
}

\author{
M. Kunert-Bajraszewska ${ }^{1}$, K. Katarzyński ${ }^{1}$, and A. Janiuk ${ }^{2}$ \\ 1 Toruń Centre for Astronomy, Faculty of Physics, Astronomy and Informatics, NCU, Grudziądzka 5, 87-100 Toruń, Poland \\ e-mail: magda@astro.uni.torun.pl \\ 2 Centre for Theoretical Physics, Polish Academy of Sciences, Al. Lotników 32/46, 02-668 Warsaw, Poland
}

Received 1 August 2014 / Accepted 27 November 2014

\section{ABSTRACT}

\begin{abstract}
Aims. We present modelling and interpretation of the continuum broadband emission of two broad absorption line (BAL) quasars. The X-ray weakness of BAL quasars in comparison to non-BAL objects is possibly caused by the absorption of X-ray emission by the shielding material near the equatorial plane. On the other hand, the radio-loud BAL quasars are more X-ray loud than the radio-quiet ones. This suggests that part of the X-ray emission may arise from the radio jet. To investigate this possibility, we modelled the nuclear spectra of two BAL quasars in the whole available energy range.

Methods. We focus on the emission from the very centres of these two objects, not greater than several parsecs. The source of emission was approximated by a single, homogeneous component that produces synchrotron and inverse-Compton radiation. The simplicity of the model allowed us to estimate the basic physical parameters of the emitting regions, using a universal analytic approach. Such methods have already been proposed to estimate basic physical parameters in blazars. For the first time, in a simplified form we propose this solution for quasars. In addition, we modelled the radiation spectra of the accretion disk and its corona to compare them with the jets' spectra.

Results. We find that in the case of radio and X-ray luminous high-redshift object 3C 270.1, the nuclear X-ray continuum is dominated by the non-thermal, inverse-Compton emission from the innermost parts of the radio jet. However, the radio core of the lobe-dominated PG 1004+130 is probably too weak to produce significant part of the observed X-ray emission. A large contribution from the X-ray emitting accretion disk and corona is produced in our model for a sufficiently high mass of the black hole. However, it then exceeds the observed flux. Because the large intrinsic absorption was postulated recently by the NuSTAR observations, we propose that the disk-corona component may still account for the X-rays produced in this source. This part of the spectrum must nevertheless be dominated by the X-ray jet. The results of our modelling show that the jet-linked X-ray emission is present in both strong and weak radio sources, but its fraction seems to scale with the radio jet power.
\end{abstract}

Key words. quasars: absorption lines - X-rays: galaxies - quasars: general

\section{Introduction}

Extremely fast outflows (reaching $0.3 \mathrm{c}$ ) of highly ionized plasma that are launched in the proximity of a supermassive black hole are possibly the origin of the blue-shifted broad absorption lines (BAL) in the UV part of the quasar spectrum. They have been under scrutiny for over 20 years. Together with powerful jets, they provide a very efficient mechanism for the mass transfer from the accretion disk. Traditionally, BALs are defined to have $\mathrm{C}_{\mathrm{IV}}$ absorption troughs at least $2000 \mathrm{~km} \mathrm{~s}^{-1}$ wide and are quantified by the balnicity index (BI) introduced by Weyman et al. (1991). When using this definition, the fraction of BAL quasars among the whole quasar population is only $\sim 15 \%$ (Hewett $\&$ Foltz 2003; Trump et al. 2006; Dai et al. 2008; Maddox et al. 2008; Knigge et al. 2008; Gibson et al. 2009).

Such a low value can be explained by the theoretical model of Elvis (2000) in which the outflows manifest as BALs in the quasar spectrum only if they are seen under specific, middlerange inclination angles. In the accretion disk wind model by Murray et al. (1995), the wind is launched from the disk at much lower inclination angles. Finally, the radio variability studies of

* Appendix $\mathrm{A}$ is available in electronic form at http://www . aanda.org some of the BAL quasars imply that there are polar outflows from the inner regions of a thin disk (Zhou et al. 2006; Ghosh $\&$ Punsly 2007). Nevertheless, according to the radio studies of BAL quasars (Shankar et al. 2008; Kunert-Bajraszewska et al. 2014), the majority of them have low radio luminosities and moderate orientations. The observed X-ray weakness of some of them agrees with the above findings.

Generally, the X-ray and UV properties of BAL quasars are consistent with those of unabsorbed quasars (Gallagher et al. 2006; Stalin et al. 2011), but both the X-ray and UV absorption are complex in these objects. Several other studies, however, find that after accounting for intrinsic absorption (Luo et al. 2013) or magnification of X-ray emission viewed from large inclination angles (Morabito et al. 2014), BAL quasars are X-ray weak compared to non-BAL objects. Moreover, the degree of $\mathrm{X}$-ray weakness for radio-loud and radio-quiet BAL quasars is different, with the radio-loud ones being less X-ray weak than radio-quiet ones, compared to non-BAL quasars (Miller et al. 2009). A simple scenario suggested by a few authors says that the X-ray continuum of radio-loud BAL quasars is a superposition of disk/corona and small-scale jet X-ray emission (Wang et al. 2008; Kunert-Bajraszewska et al. 2009; Miller et al. 2009).

In this work, we explore the above scenario by modelling the spectra of two radio-loud BAL quasars in the framework of 
Table 1. Basic observational parameters.

\begin{tabular}{lcccc}
\hline \hline & $3 \mathrm{C} 270.1$ & Ref. & PG 1004+130 & Ref. \\
\hline RA (J2000) & $12^{\mathrm{h}} 20^{\mathrm{m}} 33.9^{\mathrm{s}}$ & & $10^{\mathrm{h}} 07^{\mathrm{m}} 26.1^{\mathrm{s}}$ \\
Dec (J2000) & $+33^{\circ} 43^{\prime} 12^{\prime \prime}$ & & $+12^{\circ} 48^{\prime} 56^{\prime \prime}$ & \\
Redshift $z$ & 1.53 & & 0.24 & \\
$\mathrm{P}(408 \mathrm{MHz})$ & 280 & 2 & - & \\
$\mathrm{L}(1.4 \mathrm{GHz})$ & 210 & 2 & $15.8 \pm 0.4$ & 1 \\
$\mathrm{C}(5 \mathrm{GHz})$ & 190 & 2 & $27.3 \pm 0.2$ & 1 \\
$\mathrm{X}(8.4 \mathrm{GHz})$ & $107.6 \pm 0.1$ & 1 & $19.2 \pm 0.3$ & 1 \\
$\mathrm{U}(15 \mathrm{GHz})$ & $88.9 \pm 0.4$ & 1 & - & \\
$\mathrm{K}(22 \mathrm{GHz})$ & - & & $57.9 \pm 2.0$ & 1 \\
$\mathrm{X}-\mathrm{ray}$ & $6 \pm 0.22$ & 3 & $4.5 \pm 0.10$ & 4 \\
& $(0.3-8 \mathrm{keV})$ & & $(0.5-8 \mathrm{keV})$ & \\
photon index $\gamma$ & 1.66 & 3 & 1.50 & 4 \\
$\log L_{\mathrm{bol}} / L_{\mathrm{Edd}}$ & 0.34 & 5 & 0.09 & 6 \\
$\log M_{\mathrm{BH}} / M_{\odot}$ & 9.10 & 5 & 9.30 & 6 \\
$\mathrm{BI}$ & 52.5 & 7 & 850 & 8 \\
\hline
\end{tabular}

Notes. The radio and X-ray fluxes are related to the radio core $C$ (Fig. 1) and are given in the units of mJy and $10^{-13} \mathrm{erg} \mathrm{s}^{-1} \mathrm{~cm}^{-2}$, respectively. The Balnicity index (BI) is given in the units of $\mathrm{km} \mathrm{s}^{-1}$.

References. (1) this paper; (2) Stocke et al. (1985); (3) Wilkes et al. (2012); (4) Miller et al. (2006); (5) Shen et al. (2010); (6) Luo et al. (2013); (7) Gibson et al. (2009); (8) Wills et al. (1999).

an optically thick disk plus corona model and the emission of a jet. Both these quasars, PG 1004+130 and 3C 270.1, are classified as large-scale (linear size $>20 \mathrm{kpc}$ ), radio-loud active galactic nuclei (AGNs), and their radio structures are well resolved with VLA, showing central component, radio core, and jets or lobes, on both sides. Their rather symmetric morphologies suggest a some line-of-sight beaming in the jets and cores, which is an important property when interpreting the results of our modelling. In the case of PG 1004+130, the angle between the observer and the jet axis has been estimated to be $\gtrsim 45^{\circ}$ (Wills et al. 1999), and the radio core is weak in this source. Quasar 3C 270.1 is a core-dominated object. The Chandra X-ray observations also resolved the structure of both quasars. The X-ray and radio properties of the outer, kiloparsec-scale jets, together with jet spectral-energy-distribution (SED) modelling, has been described in detail by Miller et al. (2006) and Wilkes et al. (2012). Here we focus on the central regions of both sources, where most of the X-ray emission is concentrated.

\section{Multi-wavelength data}

We collected the available radio, IR, optical, and X-ray data from the literature and from the Sloan Digital Sky Survey (SDSS) database for both PG 1004+130 and 3C 270.1 quasars. The IR and optical data of both host galaxies can be found in the NASA/IPAC Extragalactic Database (NED). The Spitzer/IRAC $3.6,4.5,5.8,8,16$, and $24 \mu \mathrm{m}$ flux densities of 3C 270.1 were taken from Drouart et al. (2012). NuSTAR X-ray observations come from the work of Luo et al. (2013). However, the radio and Chandra X-ray fluxes in our modelling refer only to the central part of each quasar - the radio core. The radio and X-ray core fluxes are gathered in Table 1 for convenience. The $8 \mathrm{GHz}$ flux density of 3C 270.1 (project AB796) and the $5 \mathrm{GHz}$ (project AK298), $8 \mathrm{GHz}$ (project AW249), and $22 \mathrm{GHz}$ (project AP210) flux densities of PG 1004+130 were measured in VLA images produced by the VLA data calibration pipeline in AIPS. The $1.4 \mathrm{GHz}$ core flux density of PG $1004+130$ was measured in the Faint Images of the Radio Sky at Twenty-cm

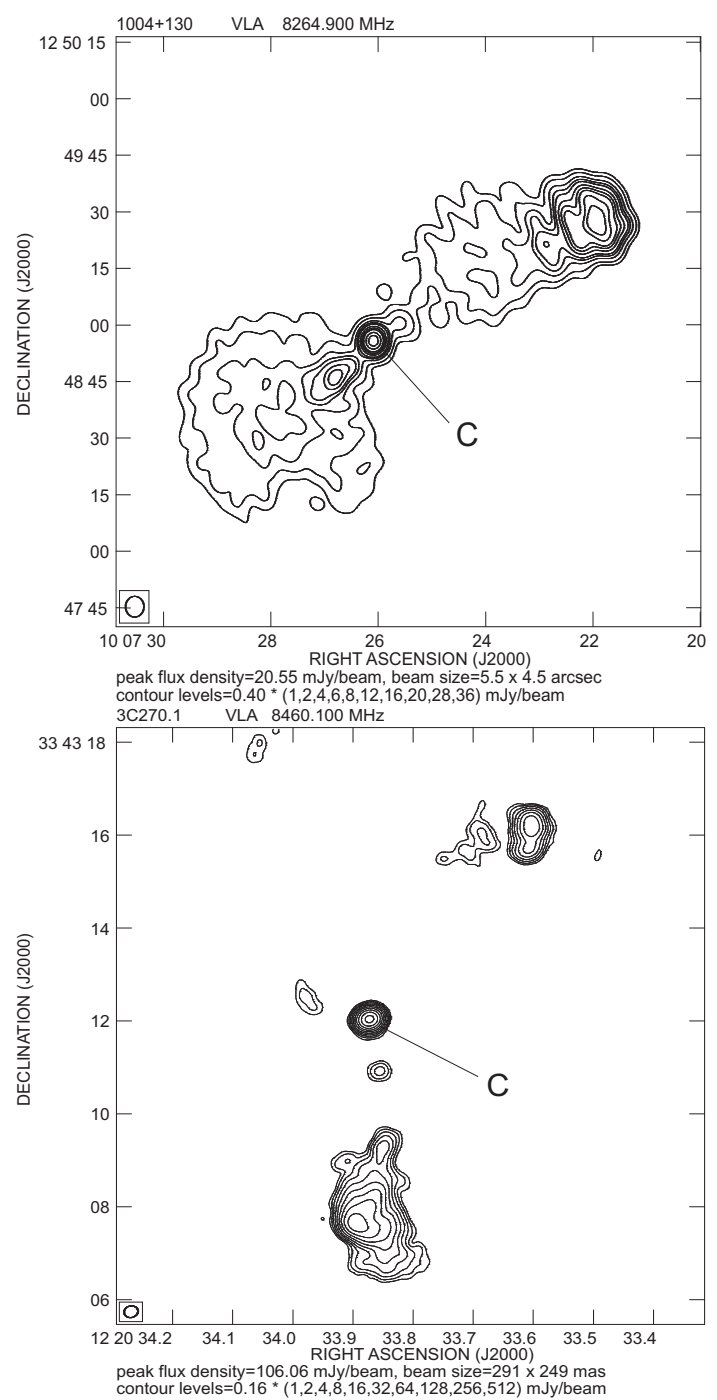

Fig. 1. VLA X-band images of PG 1004+130 (top) and 3C 270.1 (bottom). Contours increase by a factor 2 , and the first contour level corresponds to $\approx 3 \sigma$.

(FIRST) image. The final $8 \mathrm{GHz}$ images of both PG 1004+130 and 3C 270.1 quasars are presented here in Fig. 1, where the radio core is indicated as $C$.

Throughout the paper, we have assumed cosmological parameters $H_{0}=71 \mathrm{~km} \mathrm{~s}^{-1} \mathrm{Mpc}^{-1}, \Omega_{\mathrm{M}}=0.27, \Omega_{\Lambda}=0.73$.

\section{Notes on selected objects}

$P G 1004+130$. This is a low-redshift $(z=0.24)$, BAL quasar (Wills et al. 1999) with a hybrid FR I/FR,II radio morphology (Gopal-Krishna \& Wiita 2000). The south-eastern FR I-like part of the source consists of a faint lobe and a jet, which progressively fades away from the nucleus $C$. The north-western part is dominated by strongly edge-brightened radio lobe, which is typical of the FR II sources (Fig. 1), which is also the brightest component of the whole source. The overall linear size of the source is $\sim 406 \mathrm{kpc}$. The Chandra observations revealed X-ray emission aligned with the FR I jet and X-ray absorption variability (Miller et al. 2006). Most of the X-ray flux is associated with the radio core. Recently, Luo et al. (2013) have reported detecting this source in the softer NuSTAR band. 
3C 270.1. This is a high redshift $(z=1.53)$, radio-loud BAL quasar (Gibson et al. 2009). It has been classified as a "dogleg" or bent quasar (Stocke et al. 1985). The radio structure consists of a strong radio core and two jets or lobes, typical of the FR II morphology (Fig. 1). The overall linear size of the source is $\sim 80 \mathrm{kpc}$. The VLBA 8.4 and $15.4 \mathrm{GHz}$ observations resolve the radio core into one-sided core-jet structure (Hough et al. 2002). The Chandra observations of 3C 270.1 revealed compact X-ray emission of the nucleus and extended X-ray emission associated with the southern radio lobe of 3C 270.1. The extended X-ray emission probably originates in the double hotspot within that lobe and is consistent with the inverse-Compton process (Wilkes et al. 2012).

\section{Estimates of the physical parameters}

To estimate the basic physical parameters of the jet emission, we adopted the approach proposed already for blazars (e.g., Bednarek \& Protheroe 1997; Tavecchio et al. 1998; Katarzynski 2012). In this approach the estimations are based on specific features observed in the spectra. For example, the position of a maximum in the synchrotron emission is frequently used as one of the spectral features. The quality of the estimations depends on the available observations and is usually problematic. The input parameters derived from the observations have relatively large uncertainties. This is somehow compensated for by different methods used for the estimations. The procedure is reasonable if all the methods lead to similar values of the physical parameters. Finally, the estimations also depend on specific assumptions in the emission model.

We assume that some fraction of the emission of the innermost regions of BAL quasars is produced by a relatively small (a few pc or less) region of a jet. Therefore, to estimate the physical parameters of such a region, we adopt a classical scenario where relativistic electrons produce synchrotron and inverse-Compton radiation. The seed photons for the inverse-Compton scattering come from the synchrotron emission, as it does in the classical synchrotron self-Compton (SSC) process. For the sake of simplicity we assume that the emitting region is spherical $(R-$ radius) and homogeneous. The region is filled uniformly by the electrons and tangled magnetic filed ( $B$-intensity). The particle energy distribution is described by a simple power-law function $N(\gamma)=K \gamma^{-n}$, where $1 \leq \gamma \leq \gamma_{\max }$ and the energy is $E=\gamma m_{\mathrm{e}} c^{2}$. The function $N$ gives the number of particles per unit volume at a given energy. In principle, the source may travel with a relativistic velocity that implies the Doppler factor $\delta>1$. However, in all our estimates, we assume that $\delta=1$, because the superluminal motions of jet's knots were never observed in BAL quasars.

The model is described by only five free parameters $\left(R, B, K, n, \gamma_{\max }\right)$. Using the well known relation $\alpha=(n-1) / 2$, we may estimate the slope of the particle energy distribution $(n)$ from the spectral index $(\alpha)$ of the synchrotron radiation $\left(F_{\mathrm{s}} \propto\right.$ $\left.v^{-\alpha}\right)$. The SSC emission presented in $v F(v)$ plots usually shows two characteristic peaks. The first is due to the synchrotron emission and the second created by the inverse-Compton scattering. Using the position of the synchrotron peak $\left(v_{\mathrm{s}, \mathrm{p}}\right)$ and the level of the emission $\left(v F_{\mathrm{s}}\left(v_{\mathrm{s}, \mathrm{p}}\right)\right)$ at this peak, we may derive a simple relation between the magnetic field $(B)$ and the particle density $(K)$ inside the source. An independent but similar relation can be obtained from the position $\left(v_{\mathrm{c}, \mathrm{p}}\right)$ and the emission level $\left(v F_{\mathrm{c}}\left(v_{\mathrm{c}, \mathrm{p}}\right)\right)$ of the IC peak. Two additional relations between $B$ and $K$ were derived from the estimated value of the synchrotron self-absorption frequency $\left(v_{\mathrm{s}, \mathrm{a}}\right)$ and from the assumption about the equipartition between the magnetic field energy density and the particle energy density. Finally, the fifth relation that only constrains the magnetic field value was derived from the relative position of the peaks $\left(v_{\mathrm{s}, \mathrm{p}}\right.$ vs. $\left.v_{\mathrm{c}, \mathrm{p}}\right)$. Detailed explanations of these estimations and derived formulae are given in Appendix A.

\section{1. $P G 1004+130$}

To estimate the physical parameters of $1004+130$, we collected observations from radio frequencies up to the X-ray range. In the radio domain, we focused on the emission that comes directly from the core, excluding emission from the extended structures that are visible in the radio maps. We assumed that the spectral index of the optically thin radio emission is $\alpha=0.35$, and the break due to synchrotron self-absorption appears at $v_{\mathrm{s}, \mathrm{b}}=3 \times 10^{10} \mathrm{~Hz}$. The position of this break is not well established. Therefore, we assumed an uncertainty of one order of magnitude around the selected value. This is indicated by a vertical area presented in the upper panel of Fig. 2. We consider that the IR and optical ranges are strongly dominated by thermal emission of dust and gas. Thus the position and the emission level of the synchrotron peak are not determined precisely. We assume $v_{\mathrm{s}, \mathrm{p}}=10^{13} \mathrm{~Hz}$ and $v F_{\mathrm{s}}\left(v_{\mathrm{s}, \mathrm{p}}\right)=3.16 \times 10^{-13} \mathrm{erg} \mathrm{cm}^{-2} \mathrm{~s}^{-1}$ with an uncertainty of one order of magnitude for both values. We also have to estimate the position of the IC peak. The X-ray observations indicate that this peak must be located well above the Chandra energy range. However, it is not possible to determine this peak precisely. We assume $v_{\mathrm{c}, \mathrm{p}}=6.3 \times 10^{19} \mathrm{~Hz}$ and $v F_{\mathrm{c}}\left(v_{\mathrm{c}, \mathrm{p}}\right)=5 \times 10^{-13} \mathrm{erg} \mathrm{cm}^{-2} \mathrm{~s}^{-1}$ with a big uncertainty of one order of magnitude as in the previous cases. Finally, to calculate the relations between $B$ and $K$, we have to determine the radius. The upper limit for this parameter can be obtained from the radio maps or from the observed variability time scales. However, we have no information about the variability of PG 1004+130, and the core observed on the radio maps may be significantly bigger than the region where most of the emission originates. Since there is no simple way to determine the radius, we selected the value $R=2.2 \times 10^{17} \mathrm{~cm}$ that gives a good agreement between the five independent methods and allows to select one particular value of $B=0.6 \mathrm{G}$ and $K=5 \times 10^{2} \mathrm{~cm}^{-3}$ for more precise simulations that we discuss in the next section. Also, in this case we assume one order of magnitude uncertainty in the $R$ value. The selected values with the uncertainties and the resulting estimations are presented in Fig. 2. The assumed uncertainties of the input parameters give an area instead of a simple functional relationship derived in Appendix A.

\section{2. $3 C 270.1$}

To estimate the physical parameters in $3 \mathrm{C} 270.1$, we conducted similar procedure to the one for PG $1004+130$. We chose the spectral index of $\alpha=0.5$, the self-absorption frequency $v_{\mathrm{s}, \mathrm{b}}=$ $6.3 \times 10^{8} \mathrm{~Hz}$, position of the synchrotron peak $v_{\mathrm{s}, \mathrm{p}}=2.24 \times$ $10^{11} \mathrm{~Hz}, v F_{\mathrm{s}}\left(v_{\mathrm{s}, \mathrm{p}}\right)=3.16 \times 10^{-14} \mathrm{erg} \mathrm{cm}^{-2} \mathrm{~s}^{-1}$, position of the IC peak $v_{\mathrm{c}, \mathrm{p}}=10^{19} \mathrm{~Hz}, v F_{\mathrm{c}}\left(v_{\mathrm{c}, \mathrm{p}}\right)=3.16 \times 10^{-13} \mathrm{erg} \mathrm{cm}^{-2} \mathrm{~s}^{-1}$, and the radius $R=5 \times 10^{19} \mathrm{~cm}$. For all above parameters, we assumed an uncertainty of one order of magnitude around the chosen values. The observations used for the estimations, the values of the input parameters with assumed uncertainties, and the results of the estimations are presented in Fig. 3. The main difference between this estimation and the calculations performed for PG $1004+130$ is that there is no agreement between all the used methods. 

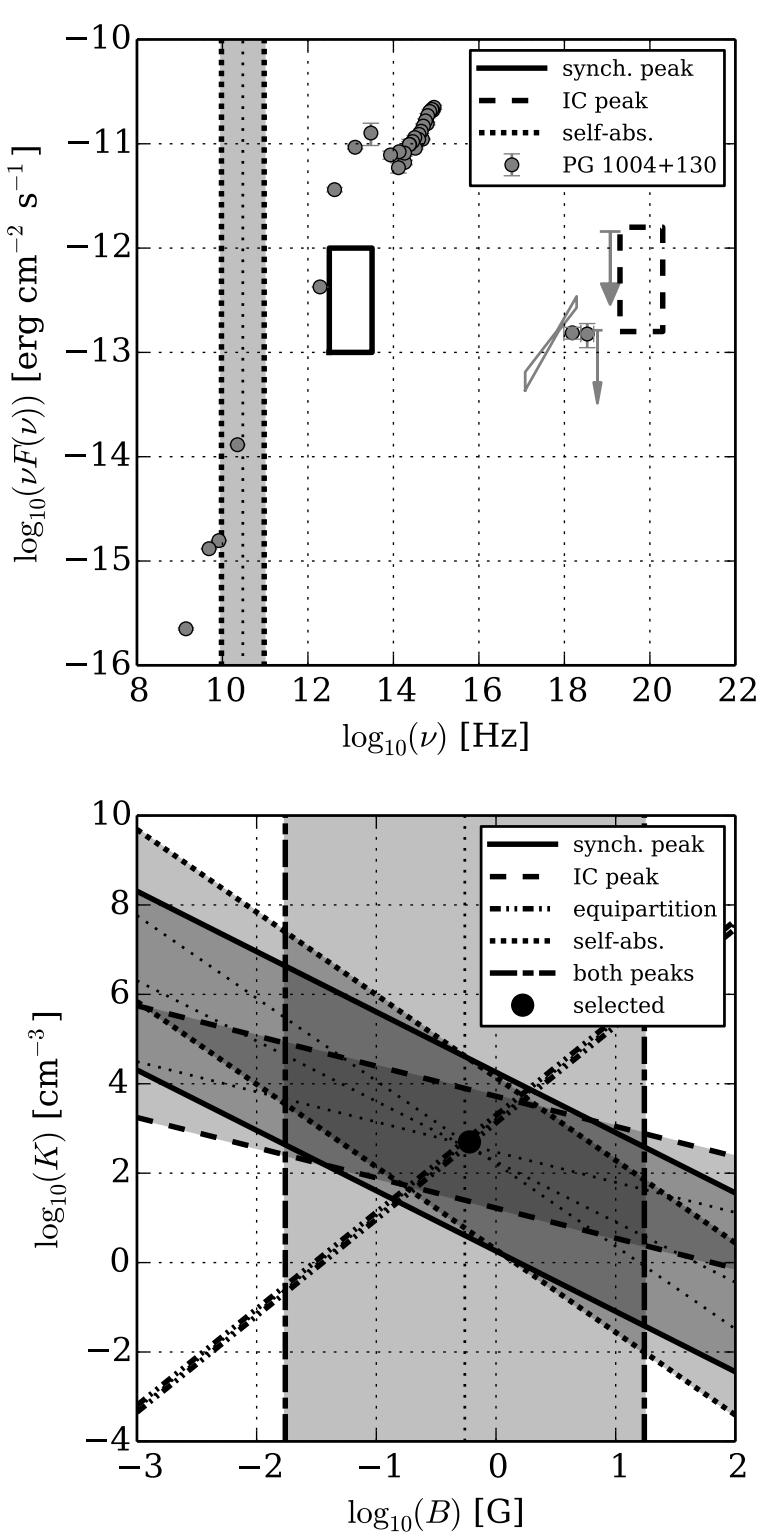

Fig. 2. Upper panel: all the observations collected to estimate physical parameters of PG $1004+130$ and the selected positions of the selfabsorption frequency, the synchrotron peak, and the IC peak with the assumed uncertainties. Lower panel: result of the estimations and the specific values $B=0.6 \mathrm{G}$ and $K=5 \times 10^{2} \mathrm{~cm}^{-3}$ (a dot) selected for the precise calculations demonstrated in Fig. 4.

The estimation that assumes equipartition between the magnetic field energy density (Eq. (A.16)) and the particle energy density (Eq. (A.17)) differs significantly from other estimations. It seems that there is no such equipartition in this particular source $\left(U_{\mathrm{e}} \gg U_{B}\right)$. This is also indicated by the fact that the IC peak is about one order of magnitude higher than the synchrotron peak. The equipartition would require $v F_{v}$ peaks at about the same level. In such a case it would be possible to obtain the agreement between the five estimation methods. However, this is not obtained. The lack of the equipartition may be caused by the efficient particle acceleration inside the emitting region. The acceleration by the first-order Fermi process at a shock wave inside the jet is frequently postulated as a source of energetic particles.
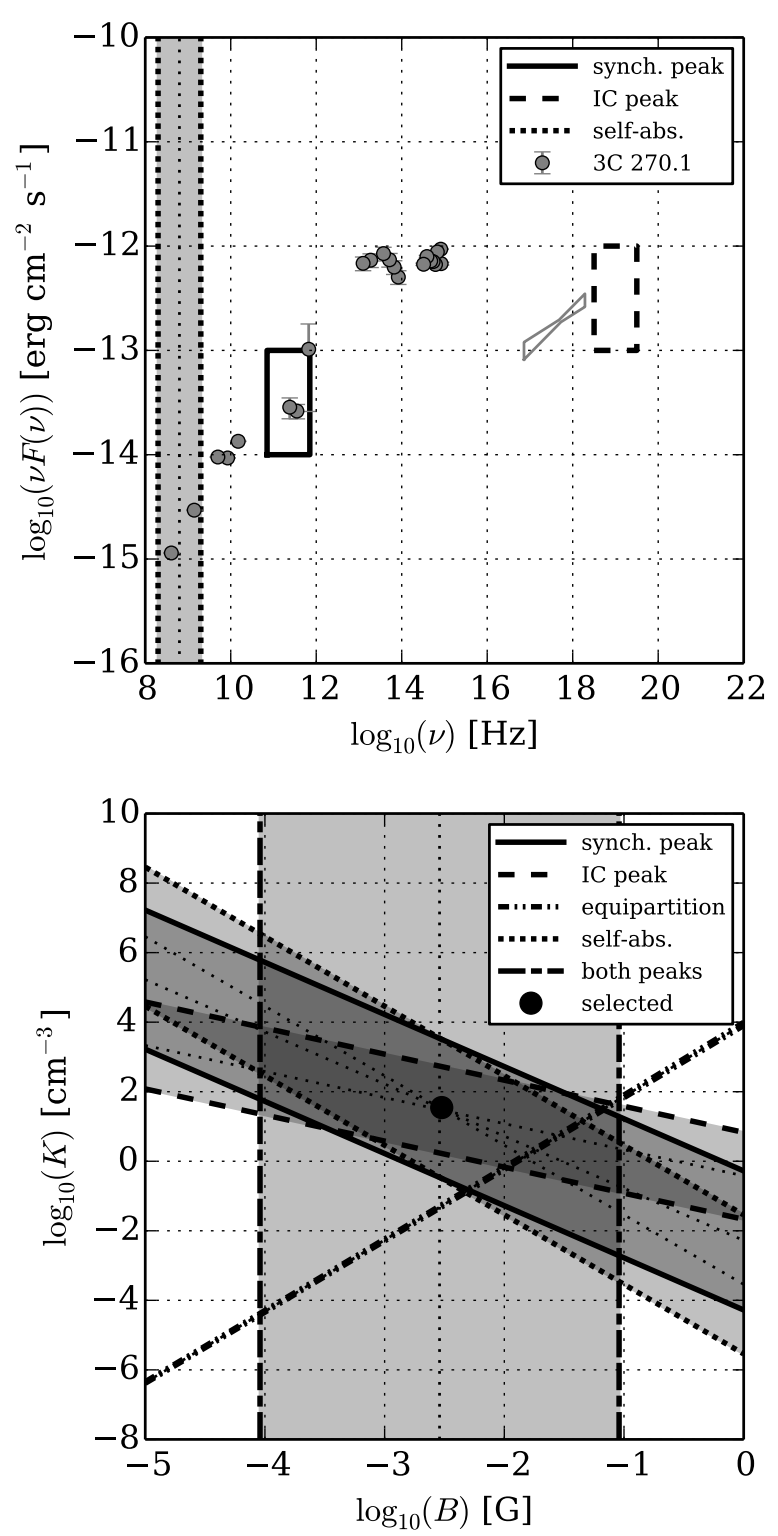

Fig.3. Upper panel: observations of 3C 270.1 collected to estimate physical parameters and the selected input parameters with the assumed uncertainties. Lower panel: result of the estimations and the specific values of $B=0.003 \mathrm{G}$ and $K=35 \mathrm{~cm}^{-3}$ (a dot) selected for the modelling presented in Fig. 5. There is no agreement between the estimation based on the equipartition and the other methods.

\section{Modelling}

We assume that most of the radio and the X-ray emission is produced by a relatively small region of a jet. Therefore, we adopt a very simple model to explain this emission. On the basis of this model, we derived five independent methods of estimating the magnetic field and the particle density inside the emitting zone. Using the estimated values and the more precise computation scheme proposed by Katarzynski et al. (2001), we show how to explain the radio and X-ray emission. To explain the emission in the IR, optical, and UV ranges, we also have to postulate other emission processes.

In the IR range the emission is probably dominated by the thermal radiation of the dust inside the host galaxy (Hughes et al. 1993; Haas et al. 1998). In principle, this kind of emission can be modelled by the grey-body spectrum. However, it appears 
necessary to assume different temperatures and emission levels for the emitting dust. Therefore, we describe this emission by

$v L(v)=v_{\mathrm{p}} L\left(v_{\mathrm{p}}\right) f(v) / f\left(v_{\mathrm{p}}\right)$,

where

$f(v)=\int_{T_{\min }}^{T_{\max }} T^{s} B_{\mathrm{gb}}(T, v) \mathrm{d} T$.

Here, $B_{\mathrm{gb}}$ is the grey-body spectrum (e.g. Kunert-Bajraszewska et al. 2009) and $T_{\min }$ and $T_{\max }$ are the minimum and maximum temperatures of the dust. The spectrum is described also by the luminosity $L\left(v_{\mathrm{p}}\right)$ at the peak $\left(v_{\mathrm{p}}\right)$ and the slope $s$ that defines the difference in the intensity of the emission. All these parameters can be derived directly from the observations.

Finally, we assume that the optical and UV emission comes mostly from the accretion disk, although the contribution from stars in the host galaxy cannot be excluded. Some fraction of the X-ray emission may come from the corona of the accretion disk. To simulate such radiation, we used the model proposed by Janiuk \& Czerny (2000). In our computations, the fraction of energy dissipated in the corona, $f_{\mathrm{c}}$, is not a free parameter but is computed self-consistently by solving a closed set of equations, and it has a value between $\sim 0.1$ and 1.0 , with the profile that is increasing with radius. Also, all the measurable quantities, i.e. the flux ratio, the spectral slopes, and the extension of the spectrum into the gamma-ray band, result from the model, including the trends for the change in these quantities with the accretion rate. The spectral shape is affected by both viscosity $\alpha_{\mathrm{d}}$ and accretion rate $\dot{m}$. In general, for a rather low viscosity, large $\dot{m}$ have an effect on the softening of the spectrum. For large $\alpha_{\mathrm{d}}$, this dependence is more complex, because the electron temperature decreases with $\alpha_{\mathrm{d}}$.

The non-rotating black hole model is taken here for simplicity, although in the case of the radio-loud quasars, the rotating black hole is usually considered. The spin of the black hole would result in a shifting of the inner radius of the disk-corona system closer to the black hole. However, the coronal emission is dominated by the outermost parts of the corona, and the X-ray spectrum would not change because of the black hole rotation. Therefore the outcome of the modelling is practically unaffected by the value of the black hole spin.

\subsection{Spectrum of $P G 1004+130$}

The radio spectrum of the core of PG 1004+130 is inverted $\alpha \simeq-0.5\left(F \propto v^{-\alpha}\right)$. This indicates that the entire radio emission is significantly self-absorbed. This requires a relatively compact source. Indeed, our estimations show that the source radius should be about $0.1 \mathrm{pc}$. What is interesting is the equipartition between $U_{B}$ and $U_{\mathrm{e}}$ in this compact object. In a more extended source, it would be impossible to obtain equipartition, because it would require many more particles to compensate for the low efficiency of the IC scattering, and therefore $U_{\mathrm{e}}$ would be much greater than $U_{B}$.

The emission from IR up to UV range can be explained by the emission of dust and the accretion disk. The IC scattering inside the jet explains the Chandra observations well (Fig. 4). Note, that there is no agreement between the Chandra and NuSTAR observations. The spectrum slope and the level of the emission are different. Therefore, we focus on the Chandra observations that seem to be more precise.

However, the theoretically obtained spectrum produced by the accretion disk and corona around a highly massive black hole

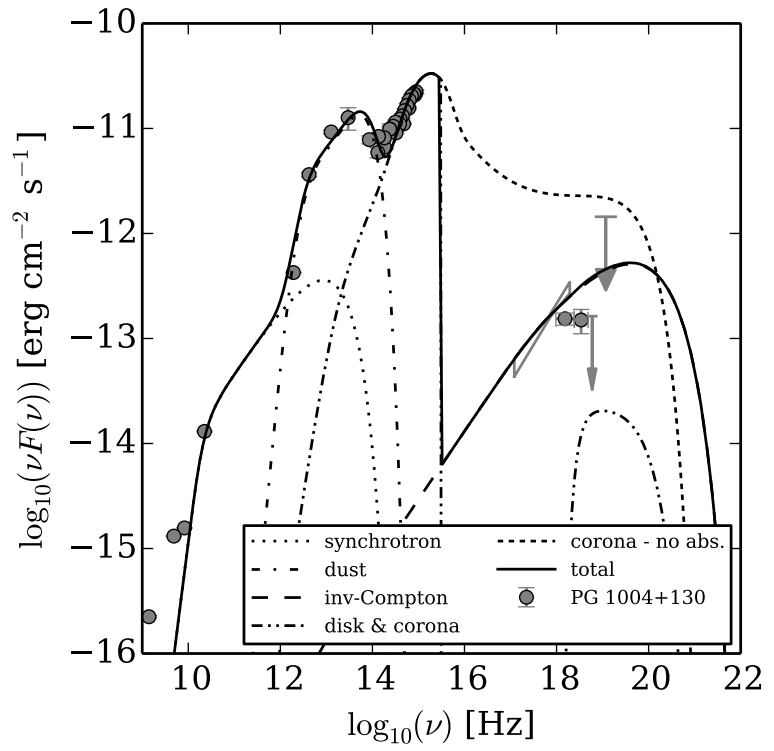

Fig. 4. Spectrum of PG 1004+130, from radio frequencies up to X-rays. To explain this spectrum it is necessary to simulate four processes: the synchrotron radiation of the jet (radio $\rightarrow$ IR), grey-body emission of dust inside the host galaxy (IR $\rightarrow$ optical), thermal emission of the accretion disk (optical $\rightarrow \mathrm{UV}$ ), and the inverse-Compton scattering inside the jet (X-rays). In addition, we add a weak hard X-ray emitting corona, which is absorbed by circumnuclear gas with a large column density on the line of sight.

(dotted blue line in Fig. 4), greatly exceeds the level of UV and $\mathrm{X}$-ray emission observed in the data for PG 1004+130. Thus, in the modelling of the disk and corona emission we consider the internal absorption of X-ray photons produced in the corona and postulated by Luo et al. (2013), based on their NuSTAR observations. As a parameter, we assumed the hydrogen column density of $7 \times 10^{24} \mathrm{~cm}^{-2}$, and we fitted the effective cross-section as a function of energy in the interstellar medium, adapted from Cruddace et al. (1974). In addition, we take the electron scattering of hard X-ray photons in the absorbing cloud into account. After these corrections, where the corona emission is absorbed, we get a satisfactory fit (Fig. 4).

\subsection{Spectrum of $3 C 270.1$}

This source appears to be significantly different from PG 1004+130. Most of the radio emission is produced by the optically thin source. The spectral index has a classical value of $\alpha=0.5$, and the self absorption seems to be significant only at the lowest observed frequencies. Therefore, the source is relatively large, a few tens of parsec. The IR and optical emission can be explained by the radiation of the dust in the galaxy. The UV emission can only be produced by the accretion disk. Since the X-ray emission is relatively high, the contribution from the corona is almost negligible. The strong X-ray flux indicates that equipartition is not possible. The parameters used to describe the spectrum of 3C 270.1 are given in Table 2, and the calculated spectra are presented in Fig. 5.

\section{Discussion}

The main aim of this paper is to explain the nuclear spectra of the selected BAL quasars in the whole observed energy range. In the literature, the origin of the X-ray emission in BAL quasars is 


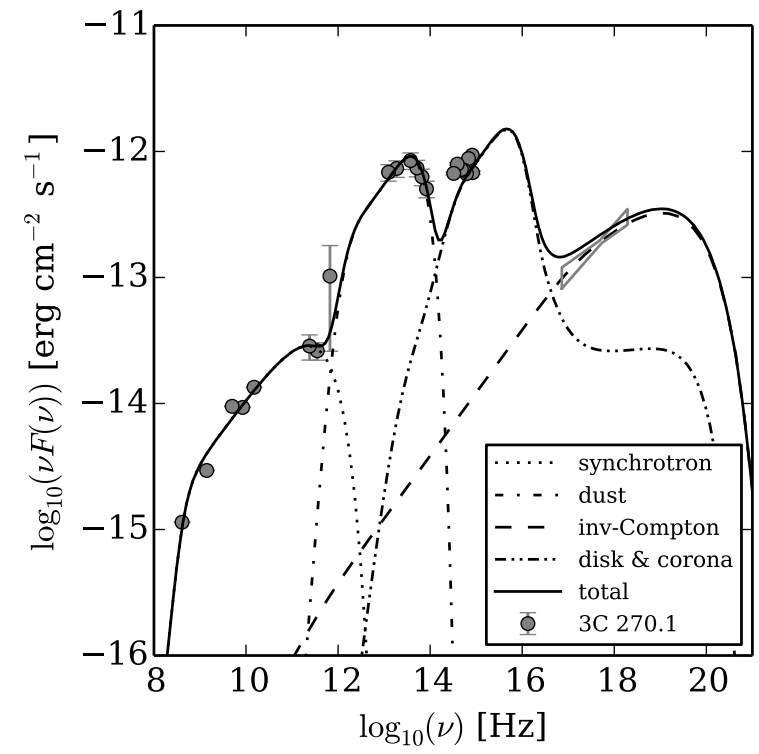

Fig. 5. Emission of 3C 270.1 in a wide frequency range calculated as the synchrotron emission of the jet (radio $\rightarrow$ IR), thermal emission of stars in the host galaxy (IR $\rightarrow$ optical), the radiation of the accretion disc and corona (UV $\rightarrow \mathrm{X}$-ray), and the IC emission generated by the jet (X-rays).

Table 2. Best fit parameters.

\begin{tabular}{lllll}
\hline \hline Params & PG $1004+130$ & $3 \mathrm{C} 270.1$ & Unit & Source \\
\hline$R$ & $2.2 \times 10^{17}$ & $5 \times 10^{19}$ & $\mathrm{~cm}$ & jet comp. \\
$B$ & 0.6 & 0.003 & $\mathrm{G}$ & jet comp. \\
$K$ & $5.0 \times 10^{2}$ & 35 & $\mathrm{~cm}^{-3}$ & jet comp. \\
$\alpha$ & 0.35 & 0.5 & & jet comp. \\
$\gamma_{\max }$ & $3.2 \times 10^{3}$ & $1.2 \times 10^{4}$ & & jet comp. \\
$\nu_{\mathrm{p}} L\left(\nu_{\mathrm{p}}\right)$ & $2.3 \times 10^{45}$ & $1.3 \times 10^{46}$ & $\mathrm{erg} / \mathrm{s}$ & dust \\
$T_{\min }$ & 50 & 45 & $\mathrm{~K}$ & dust \\
$T_{\max }$ & $1.5 \times 10^{3}$ & $2.3 \times 10^{3}$ & $\mathrm{~K}$ & dust \\
$s$ & -4.4 & -4.5 & & dust \\
$M_{\mathrm{BH}}$ & $1.2 \times 10^{9}$ & $1.7 \times 10^{9}$ & $M_{\odot}$ & disk \& cor. \\
$\dot{m}$ & 0.08 & 0.2 & $M_{\odot} / \mathrm{yr}$ & disk \& cor. \\
$\alpha_{\mathrm{d}}$ & 0.03 & 0.1 & & disk \& cor. \\
\hline
\end{tabular}

still a widely discussed issue. Given the multi-wavelength data and the interferometric radio properties of both PG 1004+130 and 3C 270.1, which are BAL quasars, it appears that the nonthermal, inverse-Compton emission from the innermost parts of the radio jet can account for a significant part of the observed X-ray emission in these objects. In the case of 3C 270.1, the jet X-ray emission is very strong, without giving the possibility of any contribution from the corona. A larger thermal corona contribution is possible in the model of less radio luminous PG $1004+130$. However, in reality PG $1004+130$ is much less X-ray luminous than 3C 270.1, which can be caused probably by the absorption of Compton-thick shielding gas (Luo et al. 2013). How are these results related to the general discussion of the X-ray properties of BAL quasars?

\subsection{X-ray properties of BAL quasars}

In the accretion disk/wind model proposed by Murray et al. (1995) and then explored by Proga et al. (2000), the outflows are launched from the accretion disk at the distance $<10^{17}$ from the supermassive black hole and radiatively driven by the UV line pressure. The X-ray emission from the innermost parts of the accretion disk and corona are absorbed interior to the UV BALs probably by the shielding gas. Its origin and geometry are still uncertain, but it is thought to be the reason for the X-ray weakness of BAL quasars. It has been suggested that the disk outflows are present in all quasars, but the effect of their presence, namely the BALs and X-ray absorption, can only be detected when looking at specific, mid-inclination angles (Elvis 2000).

Generally, after accounting for the intrinsic absorption, the $\mathrm{X}$-ray properties of BAL quasars are consistent with those of unabsorbed quasars (Gallagher et al. 2006; Stalin et al. 2011). Nevertheless, $17-40 \%$ of BAL quasars still remain X-ray weak (Luo et al. 2013). Recently, Morabito et al. (2014) have shown that when correcting for the magnification of X-ray emission via gravitational lensing by the central black hole, viewed at large inclination angles, the BAL quasars appear even more intrinsically X-ray weak. The X-ray absorption in BAL quasars is complex, often requiring fitting of an ionized or partially covering absorber, or a mixture of both. Another pure constrained issue is the relationship between UV and X-ray absorbing material. Some kind of connection is suggested by the correlation between X-ray weakness of BAL quasars and the absorption strength and maximum velocity of the UV CIV BALs (Streblyanska et al. 2010). The spectroscopic and variability studies trying to characterize the UV absorber in BAL quasars generally indicate the complex behaviour of wind outflows. BALs in radio-quiet and radio-loud quasars show depth changes in a wide range from minor to complete disappearance (Filiz et al. 2012). This can be caused, for example, by changing the location of an absorber along the line of sight (Capellupo et al. 2013). Recent observations of BAL variability in the sample of radio-loud BAL quasars support the orientation dependence of the observed outflow (Welling et al. 2014).

The radio studies of BAL quasars indicate that strong UV C IV BALs are associated with lower values of the radioloudness parameter (Welling et al. 2014; Kunert-Bajraszewska et al. 2014). Since this parameter is thought to be a good indicator of orientation (Kimball et al. 2011), its low values may imply large viewing angles and consequently the orientation dependence of BAL quasars. However, there is no correlation between the radio-loudness parameter and the BI, since a large span of values occurs in each bin of the radio-loudness parameter. Therefore, as pointed out by Kunert-Bajraszewska et al. (2014), the orientation is only one of the factors influencing absorption.

Another interesting feature of radio-loud BAL quasars is that they are less X-ray weak than radio-quiet BAL quasars compared to non-BAL objects (Miller et al. 2009). This is probably connected with the radio emission. The simple scenario suggests that the X-ray continuum in radio-loud BAL quasars consists of both accretion disk-corona and jet-linked X-ray emission coming from the innermost parts of the radio jet (Wang et al. 2008; Kunert-Bajraszewska et al. 2009; Miller et al. 2006, 2009; Berrington et al. 2013). However, the distinction of the particular components (inner and outer parts of the jet, radio core) of radio-loud BAL quasars is often impossible, because they remain unresolved even in the high-resolution observations (Jiang \& Wang 2003; Kunert-Bajraszewska \& Marecki 2007; Kunert-Bajraszewska et al. 2010; Liu et al. 2008; MontenegroMontes et al. 2008; Gawroński \& Kunert-Bajraszewska 2011; Bruni et al. 2012, 2013; Hayashi et al. 2013). The large scale BAL quasars, PG 1004+130, and 3C 270.1 discussed in this paper have clearly resolved radio structures with radio and X-ray 


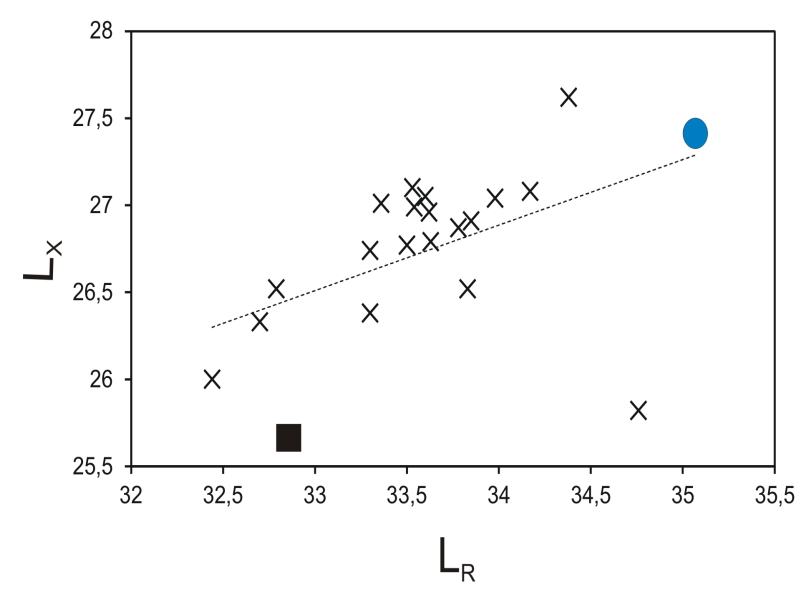

Fig. 6. X-ray - radio luminosity correlation for radio-loud BAL quasars. Data are taken from Miller et al. (2009). Luminosities are in the units of $\log \mathrm{erg} \mathrm{s}^{-1} \mathrm{~Hz}^{-1}$ at rest frame frequencies of $5 \mathrm{GHz}$ and $2 \mathrm{keV}$. BAL quasars studied in this paper are indicated with a black square (PG 1004+130) and with a blue circle (3C 270.1).

core detection. The majority of the X-ray emission is concentrated precisely in the centers of these objects.

\subsection{Two ends of the radio vs. X-ray luminosity dependence}

Both PG 1004+130 and 3C 270.1 belong to the population of BAL quasars but differ in many aspects. Their radio and X-ray luminosities place them on two different ends of the X-ray - radio dependence for radio-loud BAL quasars (Fig. 6). XMM-Newton observation of PG 1004+130 shows only minimal intrinsic absorption, while later Chandra X-ray observations of this source revealed modest X-ray absorption $\left(N_{\mathrm{H}} \sim\right.$ $10^{22} \mathrm{~cm}^{-2}$ ) with partial-covering absorbed model (Miller et al. 2006). Recently, PG $1004+130$ has been also detected in the softer NuSTAR band (4-20 keV) but not in its harder bands $(>20 \mathrm{keV})$, which may imply significant Compton-thick obscuration $\left(N_{\mathrm{H}} \sim 7 \times 10^{24} \mathrm{~cm}^{-2}\right)$. An alternative explanation of the X-ray weakness of PG 1004+130 invokes a specific mechanism that weaken the X-ray emission of the disk and corona. If the first suggestion is true, the lack of the strong $\mathrm{Fe} \mathrm{K} \alpha$ line emission is surprising and argues against the scenario in which the nucleus of PG $1004+130$ is heavily absorbed. However, the Fe $\mathrm{K} \alpha$ line emission could be diluted by the jet-linked X-ray emission (Luo et al. 2013). Such a phenomenon has already been observed in the radio-loud AGNs (Grandi et al. 2006). The brighter of our two quasars, 3C 270.1, shows no evidence of intrinsic absorption, but the $\mathrm{Fe} \mathrm{K} \alpha$ line is marginally detected (Wilkes et al. 2012).

There is also a difference between PG 1004+130 and $3 \mathrm{C} 270.1$ in the value of the BI, which quantify the UV C IV absorption. Each BAL quasar has to have BI $\left[\mathrm{km} \mathrm{s}^{-1}\right]>$ 0 , which means $\mathrm{C}_{\mathrm{IV}}$ absorption troughs that are at least $2000 \mathrm{~km} \mathrm{~s}^{-1}$ wide and at least $10 \%$ below the continuum at maximum depth (Weyman et al. 1991). The BI value amounts to 850 and 52.5 for PG 1004+130 and 3C 270.1, respectively (Wills et al. 1999; Gibson et al. 2009). As already mentioned, the strong UV absorption seems to be associated with lower values of radio-loudness parameter, $\log R_{I}<1.5$ and thus large viewing angles (Welling et al. 2014; Kunert-Bajraszewska et al. 2014). The values of the radio-loudness parameter, $\log R_{I}$, defined as the radio $(1.4 \mathrm{GHz})$-to-optical ( $i$-band) ratio of the quasar core (Kimball et al. 2011) amounts to 0.7 and 2.8 for PG 1004+130 and 3C 270.1, respectively. We can thus suspect stronger UV absorption in PG 1004+130 than in 3C 270.1.

The difference in radio properties of both quasars is also significant. 3C 270.1 is a core-dominated FR II object on VLA scales. The high-resolution 8.4 and $15.4 \mathrm{GHz}$ VLBA observations resolve the core of 3 C 270.1 into a one-sided corejet structure. Hough et al. (2002) report the presence of a strong ( $26 \mathrm{mJy}$ at $15.4 \mathrm{GHz})$ and a small ( $\sim 0.2$ mas) radio core. There is no information about the parsec scale structure of PG 1004+130, but the VLA large scale morphology is not typical. PG 1004+130 has been described as a hybrid object with two different FR structures on both sides of the source (Gopal-Krishna \& Wiita 2000). Recent studies of hybrid objects (Gawroński et al. 2006; Cegłowski et al. 2013) indicate that the interstellar medium can be responsible for their complex structures, but the properties of their central engines can also be an important factor here. Lower luminosity AGNs may develop into diffuse, large-scale structures because their weak jets are disrupted before escaping their host galaxies.

According to the analysis performed by (Willott et al. 1999), the radio luminosity of a large-scale radio source is approximately proportional to the jet power. Adopting the modifications made by (Sikora et al. 2013), we use their equation $P_{\text {jet }} \sim 10^{2} v_{1.4 \mathrm{GHz}} L_{1.4 \mathrm{GHz}}(f / 3)^{3 / 2} \mathrm{~W} \mathrm{~Hz}^{-1}$, where factor $f$ is in the range $1-20$ (we adopted $f=10$ ), to calculate jet power of the selected BAL quasars, and these amount to $10^{38} \mathrm{~W} \mathrm{~Hz}^{-1}$ and $10^{40} \mathrm{~W} \mathrm{~Hz}^{-1}$ for PG $1004+130$ and $3 \mathrm{C} 270.1$, respectively. Our modelling of the SED of both quasars indicates that the contribution of the jet-linked X-ray emission is possible in both the strong and weak radio sources, but its fraction seems to scale with jet or radio power. Since the majority of BAL quasars are weak compact radio sources without prominent jets (Bruni et al. 2012, 2013; Kunert-Bajraszewska et al. 2014), such scaling could be responsible for the difference in X-ray emission between radio-loud BAL quasars and non-BAL objects. Additionally Miller et al. (2011) suggest that beaming can increase the dominance of jet-linked X-ray emission at low inclinations in radio-loud BAL quasars.

\section{Summary}

PG 1004+130 and 3C 270.1 studied in this paper belong to the same class of BAL quasars but differ in radio, UV, and X-ray properties. We call them two ends of the radio versus X-ray luminosity correlation. They are large enough to be resolved by the high-resolution radio observations, which is rare among the BAL quasars. The clearly detected radio cores with multi-wavelength data allowed us to model their nuclear SEDs assuming that it contains both disk/corona-linked and jet-linked components. We argue that in the case of radio luminous 3C 270.1 a non-thermal, inverse-Compton emission from the innermost parts of the radio jet can account for a large amount of the observed X-ray emission. The disk/corona-linked X-ray emission is also present but is dominated by the jet-linked X-ray emission. In the case of lobe-dominated PG $1004+130$, the radio core is probably too weak to produce any significant part of observed X-ray emission. A large contribution from the X-ray emission of the accretion disk and corona is possible in our model. However, in reality the observed X-ray emission of PG 1004+130 is much weaker, and such large suppression can be caused by the absorbing gas present near the black hole. As a consequence, a large part of the X-ray emission observed in PG 1004+130 must arise in the radio jet. 
The results of our modelling show that the jet-linked X-ray emission is present in both the strong and weak radio sources, but its fraction scales with jet or radio power. Orientation could be an additional factor influencing the observed emission. It is more difficult to constrain the fraction of the disk/corona-linked $\mathrm{X}$-ray emission. It could be orientation dependent, but recent results suggest that it is a simplification and the nature, and behaviour of X-ray absorber is more complex.

Acknowledgements. We thank Bożena Czerny for discussion and constructive comments. This work was supported by the National Science Centre under grant DEC-2011/01/D/ST9/00378, and partially by grant DEC-2012/05/E/ST9/03914. The National Radio Astronomy Observatory is a facility of the National Science Foundation operated under cooperative agreement by Associated Universities, Inc. The VLA data was calibrated using NRAO's "VLA data calibration pipeline" in AIPS. This research has made use of the NASA/IPAC Extragalactic Database (NED), which is operated by the Jet Propulsion Laboratory, California Institute of Technology, under contract with the National Aeronautics and Space Administration. Funding for the SDSS and SDSS-II has been provided by the Alfred P. Sloan Foundation, the Participating Institutions, the National Science Foundation, the US Department of Energy, the National Aeronautics and Space Administration, the Japanese Monbukagakusho, the Max Planck Society, and the Higher Education Funding Council for England. The SDSS Web Site is http://www.sdss.org/. The SDSS is managed by the Astrophysical Research Consortium for the Participating Institutions. The Participating Institutions are the American Museum of Natural History, Astrophysical Institute Potsdam, University of Basel, University of Cambridge, Case Western Reserve University, University of Chicago, Drexel University, Fermilab, the Institute for Advanced Study, the Japan Participation Group, Johns Hopkins University, the Joint Institute for Nuclear Astrophysics, the Kavli Institute for Particle Astrophysics and Cosmology, the Korean Scientist Group, the Chinese Academy of Sciences (LAMOST), Los Alamos National Laboratory, the Max-PlanckInstitute for Astronomy (MPIA), the Max-Planck-Institute for Astrophysics (MPA), New Mexico State University, Ohio State University, University of Pittsburgh, University of Portsmouth, Princeton University, the United States Naval Observatory, and the University of Washington.

\section{References}

Bednarek, W., \& Protheroe, R. J. 1997, MNRAS, 292, 646

Berrington R. C., Brotherton, M. S., Gallagher, S. C., et al. 2013, MNRAS, 436, 3321

Bruni, G., Mack, K.-H., Dallacasa, D., et al. 2012, J. Phys. Conf. Ser., 372, 012031

Bruni, G., Dallacasa, D., Mack, K.-H., et al. 2013, A\&A, 554, A94

Capellupo, D. M., Haman, F., Shields, J. C., Halpern, J. P., \& Barlow, T. A. 2013, MNRAS, 429, 1872

Cegłowski, M., Gawroński, M., \& Kunert-Bajraszewska, M. 2013, A\&A, 557, A75

Cruddace, R., Paresce, F., Bowyer, S., \& Lampton, M. 1974, ApJ, 187, 497

Dai, X., Shankar, F., \& Sivakoff, G. R. 2008, ApJ, 672, 108

Drouart, G., De Breuck, C., Vernet, J., et al. 2012, A\&A, 548, A45

Elvis, M. 2000, ApJ, 545, 63

Filiz Ak, N., Brandt, W. N., Hall, P. B., et al. 2012, ApJ, 757, 114

Gallagher, S. C., Brandt, W. N., Chartas, G., et al. 2006, ApJ, 644, 709

Gawroński, M., Marecki, A., Kunert-Bajraszewska, M., \& Kus, A. J. 2006, A\&A, 447, 63
Gawroński, M. P., \& Kunert-Bajraszewska, M. 2011, PoS (X EVN Symp.), 037 [arXiv: 1106.5916]

Ghosh, K. K., \& Punsly, B. 2007, ApJ, 661, L139

Gibson, R. R., Jiang, L., Brandt, W. N., et al. 2009, ApJ, 692, 758

Ginzburg, V. L., \& Syrovatskii, S. I. 1965, ARA\&A, 3, 297

Gopal-Krishna, \& Wiita, P. J. 2000, A\&A, 363, 507

Grandi, P., Malaguti, G., \& Fiocchi, M. 2006, ApJ, 642, 113

Haas, M., Lemke, D., Stickel, M., et al. 1998, A\&A, 338, L33

Hayashi, T., Doi, A., \& Nagai, H. 2013, ApJ, 772, 4

Hewett, P. C., \& Foltz, C. B. 2003, AJ, 125, 1784

Hough, D. H., Vermeulen, R. C., Readhead, A. C. S., et al. 2002, AJ, 123, 1258

Hughes, D. H., Robson, E. I., Dunlop, J. S., \& Gear, W. K. 1993, MNRAS, 263 607

Janiuk, A., \& Czerny, B. 2000, New Astron., 5, 7

Jiang, D. R., \& Wang, T. G. 2003, A\&A, 397, L13

Katarzynski, K. 2012, 537, 47

Katarzynski, K., Sol, H., \& Kus, A. 2001, A\&A, 367, 809

Kimball, A. E., Ivezić, Ž., Wiita, P. J., \& Schneider, D. P. 2011, AJ, 141, 182

Knigge, C., Scaringi, S., Goad, M. R., \& Cottis, C. E. 2008, MNRAS, 386, 1426

Kunert-Bajraszewska, M., \& Marecki, A. 2007, A\&A, 469, 437

Kunert-Bajraszewska, M., Siemiginowska, A., Katarzyński, K., \& Janiuk, A. 2009, ApJ, 705, 1356

Kunert-Bajraszewska, M., Janiuk, A., Gawronski, M. P., \& Siemiginowska, A. 2010, ApJ, 718, 1345

Kunert-Bajraszewska, M., Cegłowski, M., Katarzyński, K., \& Roskowiński, C. 2014, MNRAS, submitted

Liu, Y., Jiang, D. R., Wang, T. G., \& Xie, F. G. 2008, MNRAS, 391, 246

Luo, B., Brandt, W. N., Alexander, D. M., et al. 2013, ApJ, 772, 153

Maddox, N., Hewett, P. C., Warren, S. J., \& Croom, S. M. 2008, MNRAS, 386, 1605

Miller, B. P., Brandt, W. N., Gallagher, S. C., et al. 2006, ApJ, 652, 163

Miller, B. P., Brandt, W. N., Gibson, R. R., Garmire, G. P., \& Shemmer, O. 2009, ApJ, 702, 911

Miller, B. P., Brandt, W. N., Schneider, D. P., et al. 2011, ApJ, 726, 20

Montenegro-Montes, F. M., Mack, K.-H., Vigotti, M., et al. 2008, MNRAS, 388, 1853

Morabito, L. K., Dai, X., Leighly, K. M., Sivakoff, G. R., \& Shankar, F. 2014, ApJ, 786, 58

Murray, N., Chiang, J., Grossman, S. A., \& Voit, G. M. 1995, ApJ, 451, 498

Proga, D., Stone, J. M., \& Kallman, T. R. 2000, ApJ, 543, 686

Shankar, F., Dai, X., \& Sivakoff, G. R. 2008, ApJ, 687, 859

Shen, Y., Richards, G. T., Strauss, M. A., et al. 2011, ApJS, 194, 45

Sikora, M., Kozieł-Wierzbowska, D., Madejski, G. M., \& Asari, N. V. 2013, ApJ, 765,62

Stalin, C. S., Srianand, R., \& Petitjean, P. 2011, MNRAS, 413, 1013

Stocke, J. T., Burns, J. O., \& Christiansen, W. A. 1985, ApJ, 299, 799

Streblyanska, A., Barcons, X., Carrera, F. J., \& Gil-Merino, R. 2010, A\&A, 515, A2

Tavecchio, F., Maraschi, L., \& Ghisellini, G. 1998, ApJ, 509, 608

Trump, J. R., Hall, P. B., Reichard, T. A., et al. 2006, ApJS, 165, 1

Wang, J., Jiang, P., Zhou, H., et al. 2008, ApJ, 676, L97

Welling, C. A., Miller, B. P., Brandt, W. N., Capellupo, D. M., \& Gibson, R. R. 2014, MNRAS, 440, 2474

Weymann, R. J., Morris, S. L., Foltz, C. B., \& Hewett, P. C. 1991, ApJ, 373, 23

Wilkes, B. J., Lal, D. V., Worrall, D. M., et al. 2012, ApJ, 745, 84

Willott, C. J., Rawlings, S., Blundell, K. M., \& Lacy, M. 1999, MNRAS, 309 1017

Wills, B. J., Brandt, W. N., \& Laor, A. 1999, ApJ, 520, L91

Zhou, H., Wang, T., Wang, H., et al. 2006, ApJ, 639, 716

Pages 9 to 10 are available in the electronic edition of the journal at http://www. aanda. org 


\section{Appendix A}

We derived four independent methods of estimating the relation between magnetic field $(B)$ and particle density $(K)$ for the simple SSC model used in this work. In addition we provide a simple method of constraining the magnetic field value.

\section{A.1. Synchrotron peak}

According to our assumption, the particle energy distribution is described by a power-law function

$N(\gamma)=K \gamma^{-n}$ for $1 \leq \gamma \leq \gamma_{\max }\left[\mathrm{cm}^{-3}\right]$.

For such a simple distribution, the synchrotron emissivity in the source's comoving frame can also be approximated by a powerlaw relation

$j_{\mathrm{s}}\left(v^{\prime}\right)=\frac{C_{\mathrm{s}}(n) K B^{\alpha+1}}{4 \pi}\left(v^{\prime}\right)^{-\alpha}\left[\frac{\mathrm{erg}}{\mathrm{s} \mathrm{cm}^{3} \text { sterad Hz}}\right]$,

where $\alpha=(n-1) / 2$ and the coefficient

$$
\begin{aligned}
C_{\mathrm{s}}(n)= & \frac{4 \sqrt{3} \pi e^{3}}{m_{\mathrm{e}} c^{2}}\left(\frac{3 e}{2 \pi m_{\mathrm{e}} c}\right)^{\frac{n-1}{2}} \Gamma\left(\frac{3 n-1}{12}\right) \Gamma\left(\frac{3 n+19}{12}\right) \\
& \times \Gamma\left(\frac{n+5}{4}\right) /(8 \sqrt{\pi}(n+1) \Gamma((n+7) / 4))
\end{aligned}
$$

(e.g. Ginzburg \& Syrovatskii 1965). The coefficient above is quite complex. However, it can be approximated very well by a simple polynomial formula

$\log _{10}\left(C_{\mathrm{s}}\right) \simeq 0.08 n^{2}+2.76 n-24.27$

that is precise enough $\left(\Delta C_{\mathrm{s}}<2 \%\right.$ for $\left.1.5 \leq n \leq 4\right)$ for our estimations. All the formulas we present in this appendix are universal, applicable for any synchrotron and/or SSC source. Therefore, our goal was to provide as simple equations as possible. In all the cases we provide a simple polynomial approximation of more complex coefficients.

The intensity of the surface emission of spherical, optically thin source is given by

$I_{\mathrm{s}}\left(v^{\prime}\right)=\frac{4}{3} j_{\mathrm{s}}\left(v^{\prime}\right) R \quad\left[\mathrm{erg} \mathrm{s}^{-1} \mathrm{~cm}^{-2} \operatorname{sterad}^{-1} \mathrm{~Hz}^{-1}\right]$,

and the flux is defined by

$F_{\mathrm{S}}(v)=\pi \frac{R^{2}}{D_{\mathrm{L}}^{2}}(1+z) \delta^{3} I_{\mathrm{s}}\left(v^{\prime}\right) \quad\left[\mathrm{erg} / \mathrm{cm}^{2}\right]$,

where: $\delta$ is the Doppler factor, $z$ the redshift, $D_{\mathrm{L}}$ the luminosity distance. The frequency transformation is given by $v=v^{\prime} \delta /(1+$ $z$ ). Using these formulae we may write our first $B \rightarrow K$ relation

$K(B)=D_{\mathrm{s}}(n) \frac{F_{\mathrm{s}, \mathrm{p}}}{f_{\mathrm{s}}}$

where

$f_{\mathrm{s}}=\frac{C_{\mathrm{s}}}{3} \frac{R^{3}}{D_{\mathrm{L}}^{2}}(1+z)^{1-\alpha} \delta^{3+\alpha} B^{\alpha+1} v_{\mathrm{s}, \mathrm{p}}^{-\alpha}$,

and

$D_{\mathrm{s}}(n)=0.61 n^{4}-5.97 n^{3}+22.2 n^{2}-38.4 n+27.6$ is the correction coefficient. We use a simple power-law approximation of the synchrotron spectrum that differs from precisely calculated spectrum, especially at the $v F_{\mathrm{s}}(v)$ peak, where the precise spectrum has a curved shape. Therefore, it is necessary to introduce an adequate correction. We have calculated the difference between the precise and the approximated spectrum for different values of $n(1.5 \leq n<3)$ and expressed this difference by the polynomial presented above. We note that $F_{\mathrm{s}, \mathrm{p}}$ and $v_{\mathrm{s}, \mathrm{p}}$ are observable quantities, the Doppler factor in all our estimations is assumed to be $\delta=1$, and the radius is constrained from the radio maps.

\section{A.2. Inverse-Compton peak}

The inverse-Compton emissivity in our simple SSC scenario can also be approximated by a power-law function

$j_{\mathrm{c}}\left(v^{\prime}\right)=C_{\mathrm{c}}(\alpha) K^{2} B^{\alpha+1}\left(v^{\prime}\right)^{-\alpha}\left[\frac{\mathrm{erg}}{\mathrm{s} \mathrm{cm}^{3} \text { sterad Hz}}\right]$,

where $\log _{10}\left(C_{\mathrm{c}}\right)=6.58 \times 10^{-4} \alpha-46.2$. This approximation is valid only in the Thompson regime of the scattering. The intensity, flux, and frequency transformations are identical as in the case of the synchrotron emission. Thus, second $B \rightarrow K$ relation is

$K(B)=\sqrt{D_{\mathrm{c}}(n) \frac{F_{\mathrm{c}, \mathrm{p}}}{f_{\mathrm{c}}}}$,

where by analogy

$f_{\mathrm{c}}=\frac{C_{\mathrm{c}}}{3} \frac{R^{3}}{D_{\mathrm{L}}^{2}}(1+z)^{1-\alpha} \delta^{3+\alpha} B^{\alpha+1} v_{\mathrm{c}, \mathrm{p}}^{-\alpha}$,

and the correction coefficient is given by

$D_{\mathrm{c}}(n)=-5.1 n^{3}+37.3 n^{2}-100.6 n+102.5$.

The other assumptions for this estimation are identical, as in the case of the synchrotron emission.

\section{A.3. Equipartition}

The other relation was derived from the assumption about the equipartition $\left(U_{B}=U_{\mathrm{e}}\right)$ between the magnetic field energy density

$U_{B}=\frac{B^{2}}{8 \pi}$

and the particle energy density

$U_{\mathrm{e}}=m_{\mathrm{e}} c^{2} \int_{1}^{\gamma_{\max }} \gamma N(\gamma) \mathrm{d} \gamma$

which gives

$K(B)= \begin{cases}\frac{B^{2}}{8 \pi m_{\mathrm{e}} c^{2} \ln \gamma_{\max }}, & n=2 \\ \frac{(2-n) B^{2}}{8 \pi m_{\mathrm{e}} c^{2}\left(\gamma_{\max }^{2-n}-1\right)}, & n \neq 2,\end{cases}$

where the Lorentz factor that characterize the maximal energy of the particles was obtained from the synchrotron peak frequency

$\gamma_{\max }=\sqrt{\frac{v_{\mathrm{s}, \mathrm{p}} \delta}{(1+z) P_{\mathrm{s}}(n) B}}$,

where $P_{\mathrm{s}}(n)=-1.9 \times 10^{5} n^{3}+8.4 \times 10^{5} n^{2}-1.9 \times 10^{6} n+3.5 \times$ $10^{6}$ was obtained for the specific case of the synchrotron peak created by an abrupt cutoff in the particle energy distribution (Eq. (A.3)). 


\section{A.4. Self-absorption frequency}

We assumed that the source is optically thin (Eq. (A.7)) at the frequencies (IR to X-ray) where the peaks are observed. This is very reasonable assumption that simplifies the calculations. However, at radio frequencies, the synchrotron self-absorption process may drastically change the slope of the spectrum. The low-frequency, self-absorbed emission will have a constant slope $\alpha=5 / 2$, whereas the spectral index of the optically thin part will depend on the particle energy slope $(\alpha=(n-1) / 2)$. This gives a characteristic break in the spectrum at the frequency that depends on $B, K, R$. The coefficient that describes the absorption for the source with a power-law particle energy distribution can also be approximated by a power law formula

$k_{\mathrm{s}}\left(v^{\prime}\right)=B_{\mathrm{s}}(n) K B^{1.5+\alpha}\left(v^{\prime}\right)^{-(\alpha+5 / 2)} \quad\left[\mathrm{cm}^{-1}\right]$

where $\log _{10}\left(B_{\mathrm{s}}\right)=0.14 n^{2}+2.43 n+6$ for $1.5 \leq n \leq 4$. Assuming that at the break frequency $\left(v_{\mathrm{s}, \mathrm{b}}\right)$ the optical depth $\left(\tau=R k_{\mathrm{s}}\right)$ is equal unity, we may obtain another relation between $B$ and $K$

$K(B)=\frac{\left((1+z) v_{\mathrm{s}, \mathrm{b}} / \delta\right)^{\alpha+2.5}}{B_{\mathrm{s}}(n) R B^{\alpha+1.5}}$

\section{A.5. Relative peak positions}

Finally, a simple constraint for only the magnetic field can be obtained from the formula (A.19)

$B=\frac{(1+z) v_{\mathrm{s}, \mathrm{p}}}{P_{\mathrm{s}}(n) \gamma_{\mathrm{max}}^{2} \delta}$

where this time $\gamma_{\max }^{2}=v_{\mathrm{c}, \mathrm{p}} /\left(P_{\mathrm{c}}(n) v_{\mathrm{s}, \mathrm{p}}\right)$ and $P_{\mathrm{c}}(n)=-0.48 n+1.3$. The maximum energy $\left(\gamma_{\max }\right)$ was derived from relative position of the peaks. According to the basic theory of the IC scattering, on average $v_{\mathrm{c}, \mathrm{p}}^{\prime} \simeq(4 / 3) \gamma_{\max }^{2} v_{\mathrm{s}, \mathrm{p}}^{\prime}$. However, our simulations show that in the case of simple power-law particle energy distribution with a sharp cut-of, $\mathrm{f}$ it is more precise to use the coefficient $P_{\mathrm{c}}$ that depends on the slope instead of the factor $4 / 3$, in the above formulae. 